OPEN ACCESS

Edited by:

Eric W. Reynolds, University of Texas Health Science Center at Houston, United States

Reviewed by:

Liam Mahoney,

Southmead Hospital, United Kingdom Anna Kistner, Karolinska University Hospital, Sweden

*Correspondence: Michal Javorka michal.javorka@uniba.sk

Specialty section: This article was submitted to Neonatology,

a section of the journa Frontiers in Pediatrics

Received: 14 January 2021 Accepted: 07 June 2021

Published: 01 July 2021

Citation: Javorka K, Haskova K, Czippelova B, Zibolen M and Javorka M (2021)

Blood Pressure Variability and Baroreflex Sensitivity in Premature Newborns-An Effect of Postconceptional and Gestational Age.

Front. Pediatr. 9:653573.

doi: 10.3389/fped.2021.653573

\section{Blood Pressure Variability and Baroreflex Sensitivity in Premature Newborns-An Effect of Postconceptional and Gestational Age}

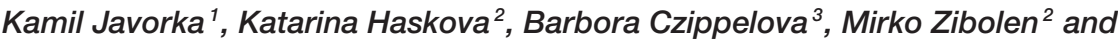 \\ Michal Javorka ${ }^{1 *}$
}

${ }^{1}$ Department of Physiology, Jessenius Faculty of Medicine in Martin, Comenius University, Martin, Slovakia, ${ }^{2}$ Clinic of Neonatology, Jessenius Faculty of Medicine and University Hospital, Martin, Slovakia, ${ }^{3}$ Biomedical Centre Martin, Jessenius Faculty of Medicine, Comenius University, Martin, Slovakia

Introduction: Cardiovascular system is the vitally important system in the dynamical adaptation process of the newborns to the extrauterine environment. To reliably detect immaturity in the given organ system, it is crucial to study the development of the organ functions in relation to maturation process.

Objectives: The objective was to determine the changes in the spontaneous short-term blood pressure variability (BPV) and baroreflex sensitivity (BRS) reflecting various aspects of cardiovascular control during the process of maturation in preterm babies and to separate effects of gestational age and postnatal age.

Methods: Thirty-three prematurely born infants without any signs of cardio-respiratory disorders (gestational age: 31.8, range: 27-36 weeks; birth weight: 1,704, range: 820-2,730 grams) were enrolled. Continuous peripheral blood pressure signal was obtained by non-invasive volume-clamp photoplethysmography method during supine rest. The recordings of 250 continuous beat-to-beat blood pressure values were processed by spectral analysis of BPV (assessed measures: total power, low frequency and high frequency powers of systolic BPV) and BRS calculation. For each infant we also assessed systolic, diastolic and mean blood pressures, heart rate and respiratory rate.

Results: With the postconceptional age, BPV measures decreased (for total power: Spearman correlation coefficient $r_{\mathrm{s}}=-0.345, P=0.049$; for low frequency power: $r_{\mathrm{s}}=-0.365, P=0.037$; for high frequency power $\left.r_{\mathrm{s}}=-0.349 ; P=0.046\right)$; and BRS increased significantly $\left(r_{s}=0.448, P=0.009\right)$. The further analysis demonstrated that these effects were more attributable to gestational age than to postnatal age. BRS correlated negatively with BPV magnitude $\left(r_{s}=-0.479\right.$ to $\left.-0.592, P=0.001-0.005\right)$. Mean blood pressure and diastolic blood pressure increased during maturation $\left(r_{\mathrm{s}}=0.517\right.$ and $0.537, P=0.002$ and 0.001 , respectively) while heart rate and respiratory rate decreased $\left(r_{\mathrm{s}}=-0.366\right.$ and $-0.516, P=0.036$ and 0.002 , respectively). 


\begin{abstract}
Conclusion: We conclude that maturation process is accompanied by an increased involvement of baroreflex buffering of spontaneous short-term blood pressure oscillations. Gestational age plays a dominant role not only in BPV changes but also in BRS, mean blood pressure, diastolic blood pressure and heart rate changes.
\end{abstract}

Keywords: preterm infants, blood pressure, blood pressure variability, baroreflex sensitivity, volume-clamp photoplethysmography

\section{INTRODUCTION}

Premature infants represent a specific group of individuals with different degrees of maturation. The degree of maturity/immaturity determines their prognosis, including various abnormalities in cardiovascular system (CVS) as well as subsequently increased risk of hypertension in adulthood (1-3). To reliably detect immaturity in the given system, it is crucial to study the development of the organ functions in the dynamics of time.

The immaturity of CVS and its control as one of the most vitally important physiological systems can be potentially risky for a premature newborn (4). Although an information on the maturation related changes in heart rate and heart rate variability reflecting heart rate control by autonomic nervous system are well-know $(5,6)$, the data focusing on another aspects of cardiovascular control - short-term blood pressure variability (BPV) and baroreflex sensitivity (BRS) - are still very rare. It is mostly caused by the methodological difficulties and unavailability associated with continuous non-invasive blood pressure recording essential for this kind of analysis (7).

Short-term BPV is a result of complex regulation of cardiovascular system, including high-pressure baroreflex, sympathetic modulation of vasculature, regulation of cardiac activity, as well as changes in venous blood return (e.g., caused by respiration) $(8,9)$. Knowledge of short-term BPV and BRS can potentially provide novel insight on maturation of the cardiovascular system and its control in premature infants.

The aim of this study was to study developmental changes in BPV and BRS in preterm infants. We addressed also the hypothesis that baroreflex buffers the blood pressure oscillations and therefore in the next step we focused on the relation between $\mathrm{BPV}$ and BRS.

\section{MATERIALS AND METHODS}

\section{Subjects}

A total of 33 children (15 boys and 18 girls) born in the 27 th -36 th gestational week with a birth weight of $820-2,730 \mathrm{~g}$ were recruited for the study. From this basic group, one child was classified as extremely preterm (below 28 weeks of gestational age at birth) and 14 were classified as very preterm (gestational age 28-32 weeks). At the time of examination, the children had a postconceptional age (gestational + postnatal age) of 32.338.4 weeks. To analyze the influence of growth after birth and deviation of birth or current weight from average values for given age we found $\mathrm{z}$-scores (distance from population mean expressed in standard deviations) for both birth and current weights. Growth after birth was quantified as a difference between $\mathrm{z}$-scores for current and birth weights.

Criteria for inclusion of newborns included postnatal age higher than 4 days and a wrist circumference of $45-75 \mathrm{~mm}$. The infants at the time of examination were without any symptoms of respiratory or cardiovascular disorders and they did not take drugs that could affect the cardiorespiratory system. Only children who were calm during the examination were included in the study. The child's motor restlessness was one of the exclusion criteria. Other exclusion criteria included respiratory or cardiovascular disorders and chromosomal abnormalities. The infants requiring phototherapy due to hyperbilirubinemia or analgosedation at the time of measurement were also excluded from our study.

The study was approved by the Ethics Committee of the Jessenius Faculty of Medicine, Comenius University in Martin (Slovakia). Written informed consent to the infant's participation in the study was given for each child by a parent or legal guardian.

\section{Data Acquisition}

Examination of children and data recording were performed between 8 a.m. and 3 p.m, 1-2 h after feeding. The measurement conditions, including environmental, were standardized with a reduction of visual and acoustic stimuli, constant ambient temperature set according to the gestational age of the child in the incubator or on a warming mattress. During recordings the children were in the supine position.

A Portapres instrument (FMS, Netherlands) was used for non-invasive and continuous peripheral blood pressure (BP) recording. $\mathrm{BP}$ registration was preceded by a selection of a cuff of the appropriate size according to the circumference of the newborn's wrist. After placing the cuff on the wrist of the right hand, which was supported and kept at heart level, the child was left at rest for at least $5 \mathrm{~min}$. Subsequently, we recorded resting beat-to-beat $\mathrm{BP}$ values during 2-5 min. Recordings of blood pressure were stored in the device memory, transferred to a computer and processed by original FMS software. During each measurement, we recorded the child's behavior and the current oxygen saturation of blood with a pulse oximeter (Nellcor Oximax N560, USA). The average value of oxygen saturation in the whole study group was $97 \%$ (range 93-100\%). Respiratory rate was measured visually from chest and abdomen movements at $30 \mathrm{~s}$ intervals.

Each child was connected to a monitor (Carescape V100 Dinamap Critikon, USA) for intermittent determination of systemic BP using oscillometric method. Two BP values 
were obtained before Portapres was used, two values after measurement by Portapres. For each infant, the oscillometrically measured BP was determined as an average of these four values.

\section{Data Processing}

From each recording, a stationary part consisting of 250 continuous beat-to-beat values of systolic BP (SBP), diastolic BP (DBP), mean BP (MBP) values and pulse intervals were found. The mean values of SBP, DBP, MBP and heart rate (HR; calculated as a reciprocal value of pulse interval) for each newborn were calculated. The spontaneous variability of SBP was analyzed by spectral analysis using fast Fourier transform (FFT) after resampling at $4 \mathrm{~Hz}$ and detrending. Window size was set at 256 resampled values. Total spectral power (TP) and spectral power in the low frequency band (LF: 0.04$0.15 \mathrm{~Hz}$ ) and in the high frequency band (HF: $0.4-1.5 \mathrm{~Hz}$ ) were calculated. Spectral bands for the neonatal population were defined according to Andriessen et al. (10, 11). While total power reflects overall blood pressure variability magnitude, blood pressure variability in high frequency band mainly reflects the mechanical effect of venous return changes associated with respiratory activity and respiratory sinus arrhythmia effect on duration of cardiac filling during diastole (9). Oscillations of the systolic blood pressure in low frequency band are associated with vascular control related to baroreflex activity and hence they are related to parasympathetic as well as to the sympathetic activity. Because the commonly calculated ratio of low- to high-frequency oscillations magnitude is considered a controversial parameter with unclear interpretation (12), its values were not calculated.

As the next step, BRS expressed in $\mathrm{ms} / \mathrm{mmHg}$ was calculated using the cross-correlation sequence method (or xBRS method) using original software provided with Portapres device. In more details, $10 \mathrm{~s}$ windows of simultaneous systolic blood pressure and pulse interval changes were analyzed to find positive crosscorrelation using time delays between blood pressure and pulse interval signal ranging from 0 to $5 \mathrm{~s}$. When cross-correlation value was significant at $P=0.01$, it indicated that increase in systolic blood pressure was accompanied by an increase in pulse interval pointing toward an involvement of baroreflex in given window of recording. The delay giving the highest correlation was chosen and instantaneous BRS value was estimated as the slope of relation between systolic blood pressure and pulse interval. Mean BRS for each newborn was calculated as a mean of instantaneous BRS values (average number of instantaneous BRS values used for the calculation of an individual BRS was 24). Data acquisition and processing are schematically presented in Figure 1.

\section{Statistical Analysis}

Shapiro-Wilk normality test as a test of the normal Gaussian distribution of analyzed data was used prior to statistical analysis. Two-sample $t$-test (for variables with Gaussian distribution) and non-parametric Mann-Whitney $U$-test (for variables with nonnormal distribution) were used to test significant differences between two subgroups. To determine the correlations between variables, Spearman's correlation coefficient $\left(\mathrm{r}_{\mathrm{s}}\right)$ and $P$-value associated with the given coefficient were determined.
For all statistics, we considered the $P$-values below 0.05 as the statistically significant. The results are presented as mean and standard deviation (SD).

\section{RESULTS}

The study was originally intended to analyze separately the effect of gestational age and postnatal age on BPV and BRS measures. However, infants with lower gestational age had greater instability of the respiratory and cardiovascular systems at the beginning of neonatal period. These children were examined only after the stabilization of their vital functions. It caused that children with low gestational age were usually examined later after birth (i.e., with higher postnatal age), while children with higher gestational age were examined earlier in their postnatal age. It caused strong negative correlation between gestational and postnatal age $\left(r_{s}=-0.87\right)$ violating the possibility to analyze effects of postnatal age and gestational age separately.

Taking together, when we consider the whole group, the effect of gestational age could not be separated from the effect of postnatal age and vice versa. Therefore, we decided to correlate assessed measures with postconceptional age only combining effect of both gestational and postnatal age.

In an effort to separate effect of gestational age from the effect of postnatal age, we selected from the study group two subgroups with 11 newborns in each of them. Two subgroups of children were paired according to postconceptional age of $35 \pm 1$ week. To minimize the spread of postconceptional age in two groups we excluded remaining 11 infants being too far from 35th week of postconceptional age from between-groups analysis. The first group (group 1) was a group with higher gestational age and lower postnatal age and the second group (group 2) was characterized by a lower gestational age and higher postnatal age. Both groups had almost the same current weight at the time of measurement. The basic study group and subgroups characteristics are presented in Table $\mathbf{1}$.

$\mathrm{BP}$ and $\mathrm{HR}$ values determined by the intermittent oscillometric method in the whole basic study group did not differ significantly from the values measured by Portapres presented in Table 1. SBP determined by the oscillometric method was 58.1 (6.1) $\mathrm{mmHg}$, DBP was 31.4 (8.2) $\mathrm{mmHg}$, MBP was 40.6 (7.4) $\mathrm{mmHg}$ and HR was $149.8\left(14.5 \mathrm{~min}^{-1}\right)$.

\section{Correlations of Postconceptional Age and Z-Scores of Birth and Current Weights to Basic Cardiorespiratory Measures and to Blood Pressure Variability (BPV) and Baroreflex Sensitivity (BRS)}

Postconceptional age in the group of all 33 newborns positively correlated with DBP $\left(\mathrm{r}_{\mathrm{s}}=0.537 ; p=0.001\right)$ and $\mathrm{MBP}\left(\mathrm{r}_{\mathrm{s}}=0.517\right.$; $p=0.002)$, BRS $\left(\mathrm{r}_{\mathrm{s}}=0.448 ; p=0.009\right)$, and it correlated negatively with $\mathrm{HR}\left(\mathrm{r}_{\mathrm{s}}=-0.366 ; p=0.036\right)$ and respiratory rate $\left(\mathrm{RR} ; \mathrm{r}_{\mathrm{s}}=-0.516 ; p=0.002\right)$. Postconceptional age also significantly negatively correlated with total power in systolic blood pressure (TP SBP), spectral power in low frequency band (LF SBP) and in high frequency band (HF SBP) (Figure 2). 


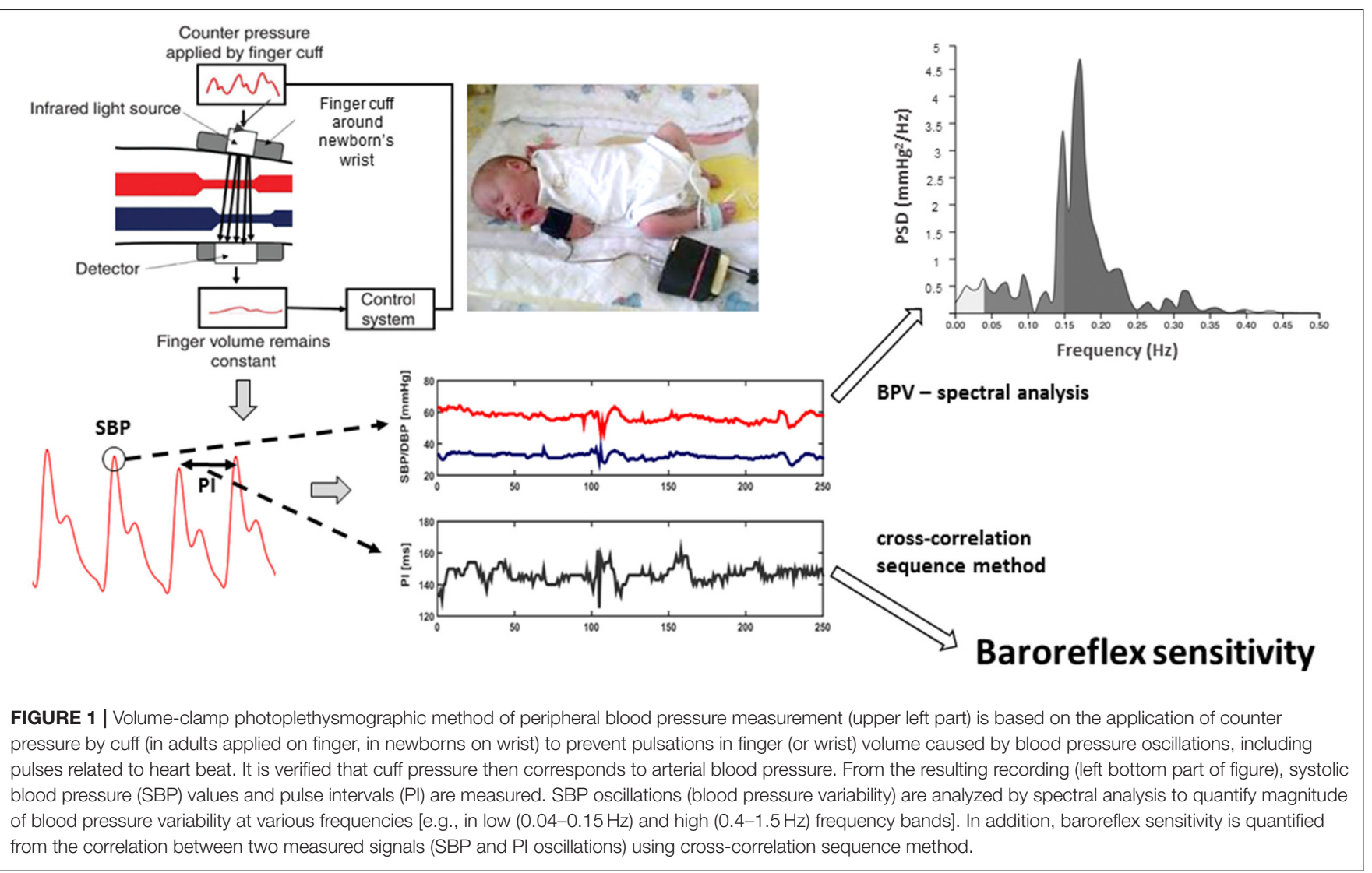

No correlation between z-scores of birth weight and current weight or of their difference with any analyzed cardiovascular measure was found.

\section{Effects of Gestational and Postnatal Age in Preterm Infants With Similar Postconceptional Age to Cardiorespiratory Measures and to Blood Pressure Variability (BPV) and Baroreflex Sensitivity (BRS)}

Despite the similar postconceptional age and current weight, the group 1 in comparison to the group 2 had higher MBP (44.0 vs. $38.0 \mathrm{mmHg}$ ), DBP (35.3 vs. $28.0 \mathrm{mmHg}$ ) and lower HR (136.9 vs. $155.7 \mathrm{~min}^{-1}$ ).

BRS was higher and the values of the HF SBP and TP SBP were significantly lower in the group 1 with longer gestational age and shorter postnatal age in comparison to the group 2 (Tables 1, 2).

\section{Baroreflex Sensitivity and Its Correlations With Blood Pressure Variability Measures and Heart Rate}

Correlations between BRS and HR and parameters of spectral analysis of SBP variability were calculated in the whole group of 33 infants. TP as well as spectral powers in LF and HF bands significantly negatively correlated with BRS (Figure 3). There was also a significant negative correlation between BRS and HR $\left(\mathrm{r}_{\mathrm{s}}=-0.594 ; P<0.001\right)$.

\section{DISCUSSION}

The most important observations of our study include the following effects of postconceptional age and gestational age on cardiovascular control in premature newborns: a decrease of blood pressure variabiliy accompanied by an increase in baroreflex sensitivity, a decrease in heart rate together with an increase of diastolic and mean blood pressures and a significant correlation between baroreflex sensitivity and blood pressure variability magnitude.

\section{Blood Pressure Variability and Baroreflex Sensitivity in Newborns}

With the introduction of noninvasive method for beat-to-beat BP recording into clinical physiology, including its application to newborns, new insight into cardiovascular control emerged. Firstly, spontaneous oscillations of BP - BPV - could be analyzed providing information related to BP control. Secondly, from the analysis of heart rate (or pulse interval) changes in relation to BP perturbations, cardiac chronotropic branch of high-pressure baroreflex characteristics including BRS can be estimated.

The mechanisms behind spontaneous BPV are complex. Similarly to heart rate variability, the blood pressure changes from heart beat to heart beat. These spontaneous changes BPV - are mostly composed of the slower (low-frequency LF) and faster (high-frequency - HF) oscillations. Slower LF oscillations in BPV of systolic blood pressure were considered 
TABLE 1 | Basic characteristics including cardiorespiratory measures of the whole study group $(n=33)$ and of two subgroups with the similar postconceptional age; values are presented as mean and standard deviation (in parentheses). Range is presented in square brackets.

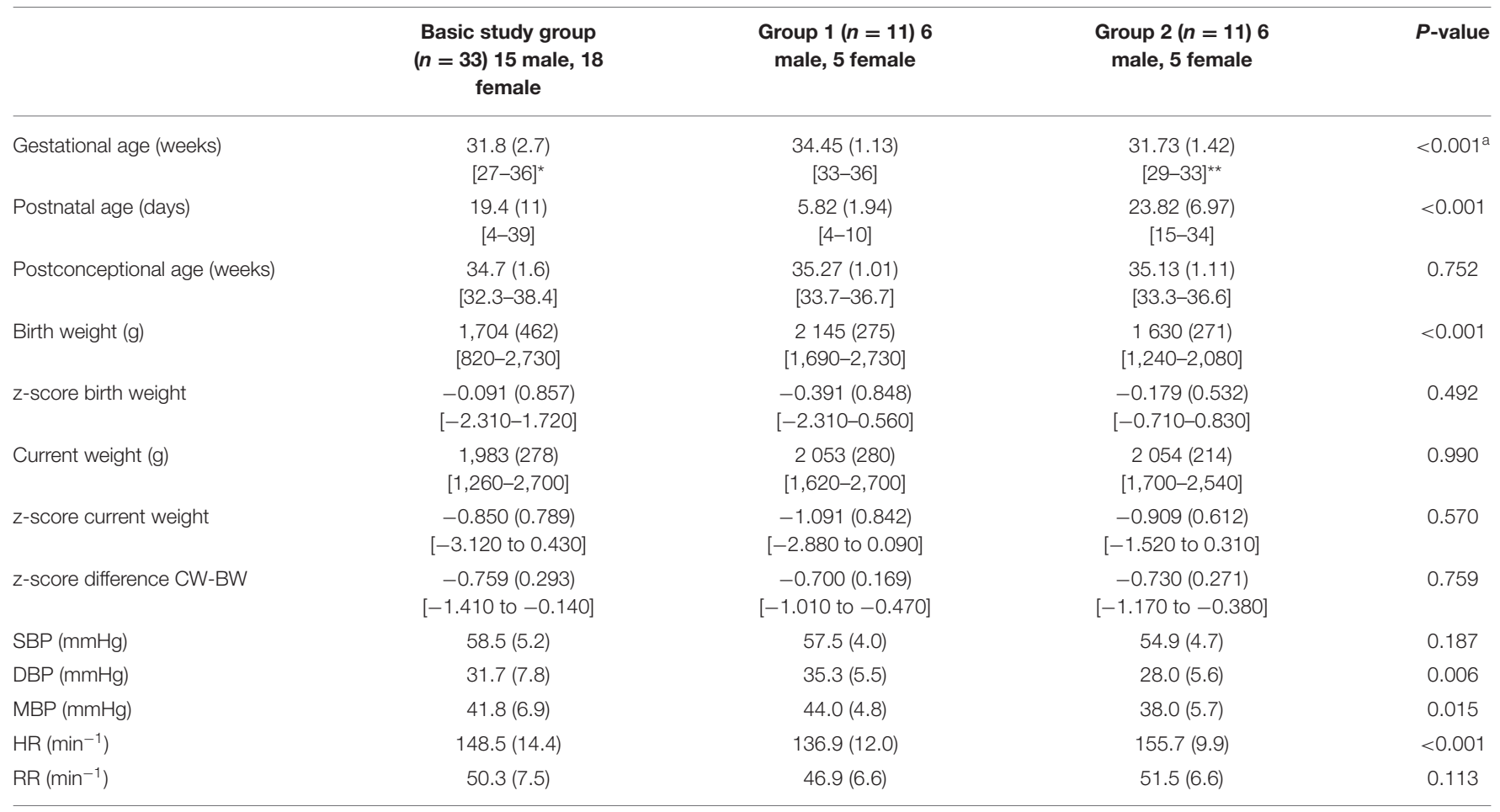

P-values correspond to between groups testing (Group 1 vs. Group 2). SBP, systolic blood pressure; DBP, diastolic blood pressure; MBP, mean blood pressure; HR, heart rate; RR,

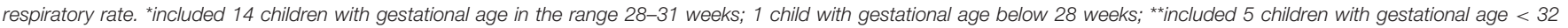
weeks. ${ }^{a}$ indicates Mann-Whitney U-test used for between groups difference testing.
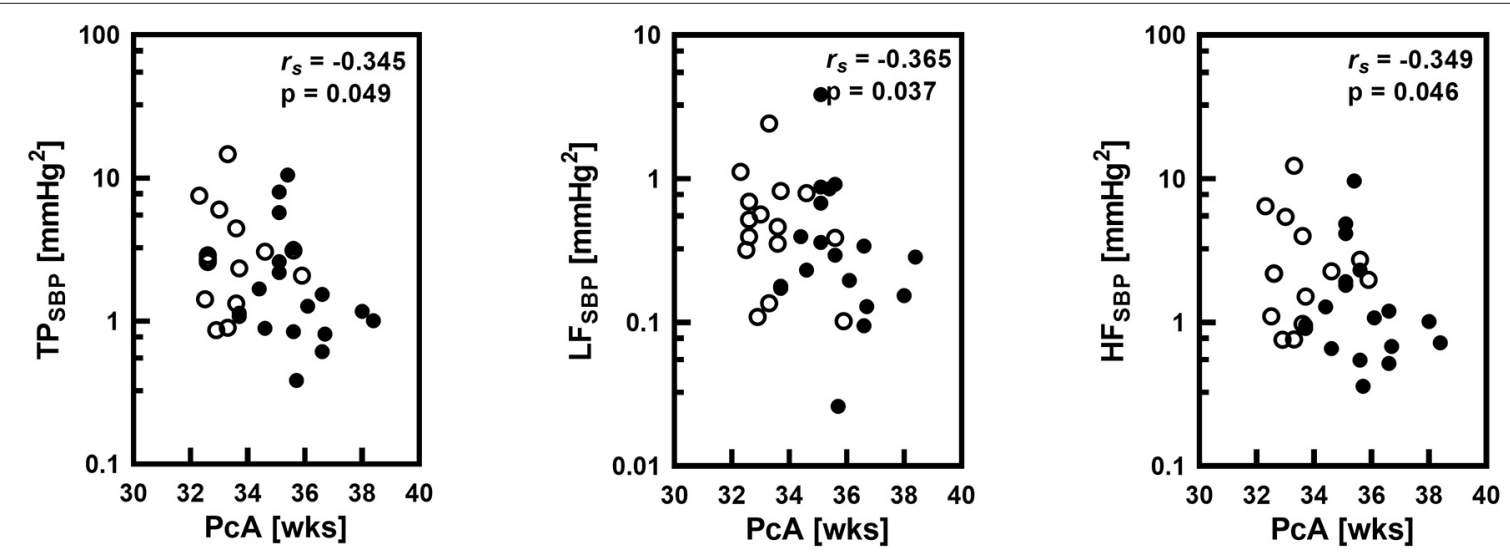

FIGURE 2 | Correlations between postconceptional age (PCA) in weeks (Wks) and spectral total power (TP), low frequency band spectral power (LF) and high frequency band spectral power (HF) of systolic blood pressure (SBP) variability with the Spearman correlation coefficient $\left(r_{\mathrm{s}}\right)$ and statistical significance level $(\mathrm{P})$. Logarithmic scale was applied on y-axis. Empty circles corresponds to infants with gestational age below 32 weeks.

to reflect mostly sympathetic activity directed to blood vessels (13). However, mechanisms behind the LF oscillations are more complex, including other mechanisms including modulation by endothelial cells lining internal surface of vessels (9) and by spontaneous changes in smooth muscle cells in vascular wall myogenic vascular control (8). In addition, a significant portion of LF SBP variability is attributable to spontaneous changes in heart rate - heart rate variability - affecting cardiac cycle length and hence DBP and SBP values (14).

SBP variability in the HF spectral band is mostly influenced by changes in cardiac contraction strength associated with ventilation (15). Cyclic intrathoracic pressure changes related to ventilation lead to changes in venous return and finally to fast cyclic changes in systolic blood pressure values. Similarly 
TABLE 2 | Comparison between two groups of newborns (Group 1 with higher gestational age, lower postnatal age; Group 2 with lower gestational age, longer postnatal age).

\begin{tabular}{lccc}
\hline & Group 1 & Group 2 & P-value \\
\hline BRS $\left(\mathrm{ms} / \mathrm{mmHg}^{2}\right.$ & $9.42(5.71)$ & $3.90(1.63)$ & 0.010 \\
LF SBP $\left(\mathrm{mmHg}^{2}\right)$ & $0.286(0.251)$ & $0.953(1.217)$ & 0.082 \\
HF SBP $\left(\mathrm{mmHg}^{2}\right)$ & $1.212(1.276)$ & $2.727(2.576)$ & 0.011 \\
TP SBP $\left(\mathrm{mmHg}^{2}\right)$ & $1.498(1.497)$ & $3.680(3.232)$ & 0.011 \\
\hline
\end{tabular}

Data are presented as the mean (standard deviation); P-value, significance level for between-groups comparison (Mann-Whitney U test). BRS, baroreflex sensitivity; LF SBP, spectral power in low frequency band; HF SBP, spectral power in high frequency band; TP SBP, total power in systolic blood pressure variability.

to LF band, venous return changes associated with heart rate variability can be also involved in the origin of HF SBP oscillations (16).

We observed the negative correlation between postconceptional age and BPV (overall BPV, as well as BPV in LF and HF bands) in the group of premature infants. It means that with a higher postconceptional age BPV in all assessed frequency bands decreases. Interestingly, when we analyzed two groups with the similar postconceptional age but different gestational and postnatal ages, we found statistically significantly lower values of TP SBP and HF SBP and a tendency toward lower LF SBP power. It indicates that the gestational age plays a dominant role in the gradual decrease of BPV in newborns.

Our results point toward the fact that the intrauterine maturation (reflected in gestational age) plays a dominant role in the stabilization of spontaneous short-term BPV, mostly in the HF band. Since blood pressure changes are mediated by multiple mechanisms partially explained above, several mechanisms could be responsible for this phenomenon:

Firstly, the decrease in the baroreflex involvement in the origin of BPV oscillations mediated by sympathetic nerves directed to the vessels can be considered. However, we assume that this mechanism will affect mostly LF oscillations in BPV and we consider this mechanism as a less probable because in our study HF SBP was affected more prominently.

Secondly, when we consider both LF and HF BPV oscillations, the changes in heart rate variability could be responsible for the changes in BPV via so called feedforward mechanism where changes in cardiac cycle length are transferred to SBP changes (16). However, based on the results of previous studies the heart rate variability increases with gestational age $(5,6)$ and we observed the opposite changes in BPV making this mechanism improbable.

Thirdly, the activity and regulatory role of the autonomic nervous system is underdeveloped in premature infants making mechanical changes caused by respiration dominant in the origin of BP oscillations (17). A reduction in intrathoracic pressure changes associated with breathing as a result of an increase in lung compliance and a reduction in airway resistance (18) can be responsible for the decrease in HF SBP with postconceptional and gestational age. Decreased lung compliance together with a weakening of strength of the Hering-Breuer inflation reflex (19) could be also responsible for the negative correlation between respiratory rate and postconceptional age found in our data.

Fourth important potential mechanism of observed BPV changes associated with postconceptional and gestational age include baroreflex acting as a buffer of BP oscillations (7). Wray et al. (20) found that vagal blockade reduces BRS and increases BPV in young healthy people. Therefore, we hypothesize that increased involvement of baroreflex expressed as increased BRS should be associated with a decreased BPV also in our neonatal group.

We tried to confirm this hypothesis by the analysis of BRS from spontaneous HR and SBP oscillation. In agreement with previous studies (21-24), higher BRS was present in children with higher gestational and postconceptional age. Subgroups analysis points toward a predominance of gestational age also in this relationship. We assume that a gradual decrease of BPV with postconceptional age is associated with the gradual increase of BRS. This observation is in concert with the concept of high-pressure baroreflex as a buffer of BP perturbations (25) confirming baroreflex as very important mechanism for stability of cardiovascular system even in healthy premature infants in early postnatal life. Infants with low BRS can be thus prone to circulatory instability.

\section{Relationship Between Postconceptional or Gestational Age and Basic Cardiorespiratory Measures}

We observed a positive correlation of postconceptional age with MBP and DBP and negative correlation with heart and respiratory rate $(\mathrm{HR}, \mathrm{RR})$. In other words, the higher was the gestational age, the higher was the systemic DBP, MBP and lower HR and RR. These findings are consistent with previous results (26-29). Subgroups analysis showed that gestational age plays the dominant role in the observed changes related to postconceptional age. Interestingly, no maturation effect on SBP was observed in our study. Considering the factors influencing DBP and subsequently MBP values based on Windkessel model (30), duration of cardiac cycle (reciprocal of HR) and steepness of BP decay during diastolic phase are the most important mechanisms contributing to changes in DBP and MBP values provided that the SBP values are similar. Postconceptional age correlated negatively with HR - it would itself lead to prolongation of diastole and a decrease of DBP (and MBP) values. However, since the positive correlation was observed between DBP or MBP values and postconceptional age, it indirectly indicates a marked increase in the steepness of BP diastolic decay. It was most probably caused by an increased peripheral vascular resistance in relation to increasing postconceptional and gestational age - but this indirect finding should be confirmed by direct measurement.

Several previous studies demonstrated that regardless of gestation week at birth where marked difference in BP values are observed, BP values become similar at the postconceptional age of $42-44$ weeks $(31,32)$. In agreement with previous data, 

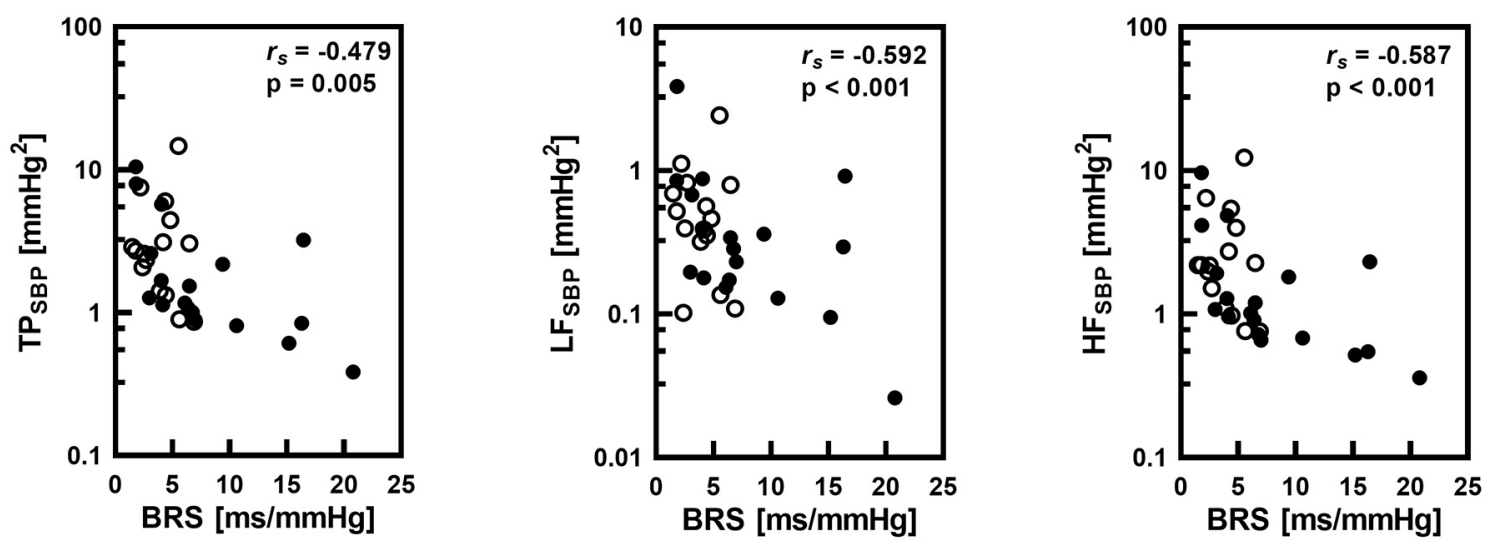

FIGURE 3 | Correlations between baroreflex sensitivity (BRS) and total power (TP), low frequency band spectral power (LF) and high frequency band spectral power (HF) of systolic pressure (SBP) variability with the Spearman's correlation coefficient $\left(r_{s}\right)$ and statistical significance level (p). Logarithmic scale was applied on $y$-axis. Empty circles corresponds to infants with gestational age below 32 weeks.

the newborns from our study born more prematurely, despite having a longer postnatal age (almost 24 vs. 6 days) at the time of measurement, have not yet been able to reach $\mathrm{BP}$ and $\mathrm{HR}$ values of babies with higher gestational and lower postnatal age at the 35 th postconceptional week. It clearly indicates that the duration of postnatal age alone in estimating the maturity of the cardiovascular system can be misleading when the gestational age is too low. We confirmed that postconceptional age in preterm infants is more accurate parameter predicting value of blood pressure than postnatal age.

In contrast, growth rate expressed as a difference in $\mathrm{z}$ scores between birth and current weights did not correlate with any analyzed measure and it did not differ between two groups. It indicates that potential effect of altered growth rate after birth did not contribute to the observed changes and relations.

\section{Methodological Limitations}

Method of the continuous non-invasive recordings of blood pressure by volume-clamp method in neonatology has great benefits but is relatively difficult and limited. The volume-clamp photoplethysmographic method (33) enables to record each beatto-beat value of SBP, MBP and DBP for analysis of spontaneous BPV together with a calculation of BRS. In newborns, the reliablity of modified method (finger cuff used in adults is applied on the wrist of newborn) for determining BP and BPV values was verified and validated by comparison with the oscillometric method as well as with the invasive intraarterial blood pressure measurement $(10,34,35)$.

The first limit stems from the size of the cuffs supplied with the device (Portapres, Finometer). It limits the use of the device to newborns with a wrist circumference in the range of $45-75 \mathrm{~mm}$, i.e., mostly to the premature newborns. A wrist circumference in our group of premature infants was in the range 49.5-74.2 mm. Using an inappropriate cuff size (e.g., smaller cuff size for a larger wrist in the full-term newborn could result in unprecise BP readings and increased discomfort of the child.
Cuff-size limitation currently restricts blood pressure variability and baroreflex analysis to premature infants - therefore it was not possible to compare premature newborns with a control group of full-term neonates to better characterize changes related to maturation.

To obtain continuous beat-to-beat recordings of systemic blood pressure in preterm infants at rest it is necessary to provide a suitable quiet and thermoneutral environment. Restlessness of a newborn, especially motoric, not only leads to physiological changes in cardiorespiratory parameters, but it can also cause artifacts by mechanical cuff compression. It could make the recording full of artifacts and hence unusable for evaluation of the spontaneous short-term BPV. In addition, although it would be interesting to observe changes in blood pressure control related to early postnatal period, due to ethical reasons the infants were examined only after stabilization of vital functions requiring $\geq 4$ days in our study group.

Regarding the measurement length, long-term inflation of the cuff on the wrist can lead to acral venous congestion and discomfort of the child. Some authors used a measurement time interval of $4-10 \mathrm{~min}$ in their studies [e.g. $(10,36,37)$ ]. Yiallorou et al. (38) used repeated 2-min episodes to register BP in premature infants. We used a measurement time of $5 \mathrm{~min}$ providing sufficiently long stationary time periods (containing 250 continuous beats) for data analysis.

Relatively small number of examined subjects associated with complex measurement procedure did not enable to account for potential sex differences and it must be also considered as a study limitation.

\section{CONCLUSIONS}

We conclude that maturation process is accompanied by a decrease in BPV and an increase of BRS reflecting buffering of $\mathrm{BP}$ changes by baroreflex. Gestational age plays a dominant role not only in BPV changes but also in BRS, MBP, DBP and HR changes. The results indicate changes related to parasympathetic 
heart rate control in relation to postconceptional and gestational age in preterm infants.

\section{DATA AVAILABILITY STATEMENT}

The raw data supporting the conclusions of this article will be made available by the authors, without undue reservation.

\section{ETHICS STATEMENT}

The studies involving human participants were reviewed and approved by Ethical Committee of the Jessenius Faculty of Medicine, Comenius University, Martin, Slovakia. Written informed consent to participate in this study was provided by the participants' legal guardian/next of kin.

\section{REFERENCES}

1. Jong de F, Monuteaux MC, van Elburg RM, Gillman MW, Belfort MB. Systematic review and meta-analysis of preterm birth and later systolic blood pressure. Hypertension. (2012) 59:226-34. doi: 10.1161/HYPERTENSIONAHA.111.181784

2. Keijzer-Veen MD, Dülger A, Dekker FW, Nauta J, Bert Vander Heijden B. Very preterm birth is a risk factor for increased systolic blood pressure at a young adult age. Pediatr Nephrol. (2010) 25:509-16. doi: 10.1007/s00467-009-1373-9

3. Yiallourou SR, Witcombe NB, Sands SA, Walker AM, Horne RSC. The development of autonomic cardiovascular control is altered by preterm birth. Early Hum Dev. (2013) 89:145-52. doi: 10.1016/j.earlhumdev.2012.09.009

4. Fyfe KL, Yiallourou SR, Horne RSC. Chapter 15: Cardiovascular consequences of preterm birth in the first year of life. In: Preterm Birth - Mother and Child. Intech Open (2012). p. 319-40. doi: 10.5772/26252. Available online at: https://www.intechopen.com/books/preterm-birth-mother-and-child/car diovascular-consequences-of-preterm-birth-in-the-first-year-of-life

5. Javorka K, Javorka M, Tonhajzerova I, Calkovska A, Lehotska Z, Bukovinska Z, et al. Determinants of herat rate in newborns. Acta Med Mart. (2011) 11:7-16. doi: 10.2478/v10201-011-0012-x

6. Longin E, Gerstner T, Schaible T, Lenz T, K?nig S. Maturation of the autonomic nervous system: differences in heart rate variability in premature vs. term infants. J Perinat Med. (2006) 34:4: 303-8. doi: 10.1515/JPM.2006.058

7. Jong de W. Blood pressure variability in neonates with a special focus on signal acquisition and signal processing. Technische Universiteit Eindhoven. (2000) 153. doi: 10.6100/IR529998

8. Höcht C. Blood pressure variability: prognostic value and therapeutic implications. ISRN Hypertension. (2013) 2013:16. doi: 10.5402/2013/398485

9. Stauss HM. Identification of blood pressure control mechanisms by power spectral analysis. Clin Exp Pharmacol Physiol. (2007) 34:3628. doi: 10.1111/j.1440-1681.2007.04588.x

10. Andriessen P, Schoffelen RLM, Berendsen RCM, de Beer NAM, Oei SG, Wijn PFF, et al. Noninvasive assessment of blood pressure variability in preterm infants. Intern Pediat Res Found. (2004) 55:220-3. doi: 10.1203/01.PDR.0000104152.85296.4F

11. Andriessen P, Janssen BJA, Berendsen RCM, Oetomo SB, Wijn PFF, Blanco CE. Cardiovascular autonomic regulation in preterm infants: the effect of atropine. Ped Res. (2004) 56:939-46. doi: 10.1203/01.PDR.0000145257.75072.BB

12. Billman GE. The $\mathrm{LF} / \mathrm{HF}$ ratio does not accuartely measure cardiac sympatho-vagal balance. Front Physiol. (2013) 4:26. doi: 10.3389/fphys.2013. 00026

13. Lazarova Z, Tonhajzerova I, Trunkvalterova Z, Brozmanova A, Honzíková $\mathrm{N}$, Javorka $\mathrm{K}$, et al. Baroreflex sensitivity is reduced in obese normotensive

\section{AUTHOR CONTRIBUTIONS}

$\mathrm{KJ}, \mathrm{MZ}$, and $\mathrm{MJ}$ designed the study. $\mathrm{KH}$ and $\mathrm{MZ}$ arranged for the probands participation. $\mathrm{KH}$ performed the measurements. $\mathrm{BC}$ and $\mathrm{KJ}$ analyzed the data and prepared figures. $\mathrm{KJ}$ and $\mathrm{MJ}$ wrote the manuscript and have contributed equally to this paper and share first authorship. KJ, MJ and MZ contributed to the interpretation of the results. KJ and MZ helped to supervise the project. All authors contributed to the article and approved the submitted version.

\section{FUNDING}

Research was supported by the grants VEGA 1/0199/19, VEGA $1 / 0200 / 19$, VEGA $1 / 0283 / 21$ and project Biomedical Center Martin ITMS code: 26220220187.

children and adolescents. Can J Physiol Pharmacol. (2009) 87:7:56571 doi: 10.1139/Y09-041

14. Porta A, Furlan R, Rimoldi O, Pagani M, Malliani A, van de Borne P. Quantifying the strength of the linear causal coupling in closed loop interacting cardiovascular variability signals. Biol Cybern. (2002) 86:24151 doi: 10.1007/s00422-001-0292-z

15. Janssen BJA, Oosting J, Slaaf DW, Persson PB, Struijker-Boudier HAJ. Hemodynamic basis of oscillations in systemic arterial pressure in conscious rats. Amer J Physiol. (1995) 269:H62-71 doi: 10.1152/ajpheart.1995.269.1.H62

16. Javorka M, Krohova J, Czippelova B, Turianikova Z, Lazarova Z, Javorka K, et al. Basic cardiovascular variability signals: mutual directed interactions explored in the information domain. Physiol Meas. (2017) 38:87794 doi: 10.1088/1361-6579/aa5b77

17. De Rogalski Landrot I, Roche F, Pichot V, Tezssier G, Gaspoy J-M, Berthelemy $\mathrm{J}-\mathrm{C}$, et al. Autonomic nervous system activity in premature and full-term infants from theoretical term to 7 years. Autonomic NeuroSci Basic Clin. (2007) 136:105-9 doi: 10.1016/j.autneu.2007.04.008

18. Battisti O, Bertrand J-M, Rouatbi H, Escandar G. Lung compliance and airway resistance in healthy neonates. Pediatr Therapeut. (2012) 2:2. doi: 10.4172/2161-0665.1000114

19. Olinsky A, Bryan $\mathrm{MH}$, Bryan AC. Influence of lung inflation on respiratory control in neonates. J Appl Physiol. (1974) 36:426-9. doi: 10.1152/jappl.1974.36.4.426

20. Wray DW, Formes KJ, Weiss MS, O-Yurvati AH, Raven PB, Zhang R, et al. Vagal cardiac function and arterial blood pressure stability. Am J Physiol Heart Circul Physiol. (2001) 281:H1870-80. doi: 10.1152/ajpheart.2001.281.5.H1870

21. Andriessen P, Oetomo SB, Peters C, Vermeulen B, Wijn PFF, Blanco CA. Baroreceptor reflex sensitivity in human neonates: the effect of postmenstrual age. J Physiol. (2005) 568:333-41 doi: 10.1113/jphysiol.2005.093641

22. Fyfe KL, Yiallourou SR, Wong FY, Odoi A, Walker AM, Horne RSC. Gestational age at birth affects maturation of baroreflex control. J.Pediat. (2015) 166:559-65. doi: 10.1016/j.jpeds.2014.11.026

23. Haskova K, Javorka M, Czippelova B, Zibolen M, Javorka K. Baroreflex sensitivity in premature infants - relation to the parameters characterizing intrauterine and postnatal condition. Physiol Res. (2017) (Suppl. 2):S25764. doi: 10.33549/physiolres.933681

24. Yiallourou SR, Sands SA, Walker AM, Horne RSC. Postnatal development of baroreflex sensitivity in infancy. J Physiol. (2010) 12:2193-203. doi: 10.1113/jphysiol.2010.187070

25. Elstad M, Toska K, Chon KH, Raeder EA, Cohen RJ. Respiratory sinus arrhythmia: opposite effects on systolic and mean arterial pressure in supine humans. J Physiol. (2001) 536 (Pt 1):251-9. doi: 10.1111/j.1469-7793.2001.t01-1-00251.x

26. Bada HS, Korones SB, Ray JD, Pourcyrous M, Magill HL, Runyan III, et al. Mean arterial pressure changes in premature infants and 
those at risk for intraventricular hemorrhage. J Pediat. (1990) 117:60714. doi: 10.1016/S0022-3476(05)80700-0

27. Lazdam M, de la Horra A, Pitcher A, Mannie Z, Diesch J, Trevitt C, et al. Elevated blood pressure in offspring born premature to hypertensive pregnancy. Is endothelial dysfunction the underlying vascular mechanism? Hypertension. (2010) 56:159-65. doi: 10.1161/HYPERTENSIONAHA.110.150235

28. Metz TD, Lynch AM, Wolfe P, Barry JS, Galan HL. Effect of small for gestational age on hemodynamic parameters in the neonatal period. J Matern Fetal Neonatal Med. (2012) 7:1979-86. doi: 10.3109/14767058.2012.671870

29. Strambi M, Vezzosi P, Buoni S, Berni S, Longini M. Blood pressure in the small -for -gestational age newborn. Minerva Pediatrica. (2004) 56:603-10.

30. Westerhof N, Lankhaar JW, Westerhof BE. The arterial Windkessel. Med Biol Eng Comput. (2009) 47:131-41 doi: 10.1007/s11517-008-0359-2

31. Lurbe E, Garcia - Vicent C, Torro I, Fayos JL,Aguilar F, De Llano, JM, et al. First-year blood pressure increase steepest in low birthweight newborns. $J$ Hypertens. (2007) 1:81-6. doi: 10.1097/HJH.0b013e32801040ec

32. Northern Neonatal Nursing Initiative. Systolic blood pressure in babies of less than 32 weeks gestation in the first year of life. Arch Dis Child Fetal Neonatal Ed. (1999) 80:F38-42. doi: 10.1136/fn.80.1.F38

33. Penaz J. Photoelectric measurement of blood pressure, volume and flow in the finger. Digest of the 10th international conference on medical and biological engineering. Dresden. (1973) 2:104.

34. Jagomägi $\mathrm{K}$, Talts $\mathrm{J}$, Raamat $\mathrm{R}$, Länsimies E. Continuous noninvasive measurement of mean blood pressure in fingers by volumeclamp and differential oscillometric method. Clin Physiol. (1996) 5:551-60. doi: 10.1111/j.1475-097X.1996.tb01020.x
35. Lemson J, Hofhuizen CM, Schraa O, Settels JJ, Scheffer G-J, van der Hoeven JG. The reliability of continuous noninvasive finger blood pressure measurement in critically ill children. Anesth Analg. (2009) 108:81421. doi: 10.1213/ane.0b013e318194f401

36. Drouin E, Gournaz V, Calamel J, Mouzard A, Rozé, J-C. Assesment of spontaneous baroreflex sensitivity in neonates. Arch Dis Child Fetal Neonatal Ed. (1997) 76:F108-12. doi: 10.1136/fn.76. 2.F108

37. Gournay V, Drouin E, Rozé JC. Development of baroreflex control of heart rate in preterm and full term infants. Arch Dis Child Fetal Neonatal Ed. (2002) 86:F151-4. doi: 10.1136/fn.86.3.F151

38. Yiallourou SR, Walker AM, Horne RSC. Validation of a new noninvasive method to measure blood pressure and assess baroreflex sensitivity in preterm infants during sleep. Neonate Sleep. (2006) 8:1083-8. doi: 10.1093/sleep/29.8.1083

Conflict of Interest: The authors declare that the research was conducted in the absence of any commercial or financial relationships that could be construed as a potential conflict of interest.

Copyright (๑ 2021 Javorka, Haskova, Czippelova, Zibolen and Javorka. This is an open-access article distributed under the terms of the Creative Commons Attribution License (CC BY). The use, distribution or reproduction in other forums is permitted, provided the original author(s) and the copyright owner(s) are credited and that the original publication in this journal is cited, in accordance with accepted academic practice. No use, distribution or reproduction is permitted which does not comply with these terms. 